\title{
Un nouveau type de co-facteur transcriptionnel impliqué dans la voie de signalisation des rétinoïdes
}

L es récepteurs nucléaires appartiennent à une famille structurellement et fonctionnellement définie de facteurs de transcription eucaryotes activés par la liaison d'un ligand. En modulant la transcription de gènes cibles en réponse à leur propre ligand et à d'autres signaux afférents, ils jouent des rôles physiologiques clés dans la régulation du développement, dans le métabolisme et dans la reproduction. Le rôle des récepteurs nucléaires activés est de diriger l'assemblage et la stabilisation du complexe de pré-initiation de la transcription dans un environnement permissif sur le promoteur d'un gène cible. Ceci implique l'interaction fonctionnelle du récepteur nucléaire avec des facteurs contenus dans ce complexe. De telles interactions sont certes nécessaires mais insuffisantes pour assurer la régulation de la transcription. Des criblages biochimiques et génétiques ont en effet permis d'identifier des protéines, les co-activateurs, qui interagissent avec les récepteurs activés et stimulent la transactivation de façon significative sans altérer l'activité transcriptionnelle basale [1,2].

Plus d'une dizaine de co-activateurs des récepteurs nucléaires ont été clonés depuis dix ans [1, 2]. Un grand nombre interagit avec le domaine AF-2 de fixation du ligand présent dans les récepteurs, par l'intermédiaire de séquences peptidiques LXXLL ou LXXLI (L: leucine; X: acide aminé quelconque; $\mathrm{I}$ : isoleucine) $\left(\mathrm{m} / \mathrm{s} 1997, n^{\circ} 10, p .1212\right)$, et ont la capacité d'activer la transcription lorsqu'ils sont recrutés à proximité d'un site de démarrage de la transcription. Ils peuvent aussi avoir d'autres fonctions comme le recrutement des facteurs de la machinerie transcriptionnelle, une activité histone acétyl transférase et donc de remodelage de la chromatine, ou être impliqués dans des interactions protéine/protéine. De plus, un rôle fonctionnel pour un ARN messager dans l'activation transcriptionnelle par les récepteurs nucléaires vient d'être identifié pour la première fois $\left(\mathrm{m} / \mathrm{s}\right.$ 1999, $\left.n^{\circ} 10, p .1153\right)$. Cet ARN appelé SRA (pour steroid receptor RNA activator) pourrait agir comme un adaptateur nucléique entre le co-activateur SRC-1 et les récepteurs des hormones stéroïdiennes. Enfin, le modèle de la régulation transcriptionnelle par les récepteurs nucléaires hormonaux apparaît converger vers le recrutement, par les récepteurs activés, de complexes et de sous-complexes composés de plusieurs polypeptides dont certains sont communs à différents facteurs de transcription. Ces complexes appelés TRAP (thyroid hormone associated receptor), ARC (activator recruted cofactors), ou encore DRIP (vitamin $D_{3}$ receptor interacting protein) semblent agir directement, après l'étape de remodelage de la chromatine, au niveau du complexe de préinitiation de la transcription [3].

L'étude de la voie d'activation des rétinoïdes nous a permis d'identifier un co-facteur spécifique de cette voie dont la particularité est de se lier à la fois aux récepteurs et aux ligands.

La voie de signalisation de l'acide rétinoïque : récepteurs nucléaires et autres protéines fixant le ligand

L'acide rétinoïque (AR), métabolite actif de la vitamine A, est un puissant modulateur de la croissance et de la différenciation cellulaire et joue un rôle central dans les processus de développement de l'embryon et dans l'homéostasie des tissus adultes. Si, dès les années 1960, certains éléments des syndromes de carence en vitamine $\mathrm{A}$ ont $\mathrm{pu}$ indiquer son rôle dans l'hématopoïèse, celui-ci n'a été étudié que dans les années 1980, tout d'abord dans la différenciation des cellules leucémiques puis dans l'hématopoïèse normale. La plupart des effets biologiques de l'acide rétinoïque sont relayés par deux familles de récepteurs nucléaires, les RAR (retinoic acid receptor) dont les ligands sont l'acide rétinoïque tout-trans et 9cis, et les RXR (retinoid $X$ receptor) qui ne lient que l'acide rétinoïque 9-cis. RAR et RXR appartiennent tous deux à la superfamille des récepteurs des stéroïdes/rétinoïdes/hormones thyroïdiennes et de la vitamine D3 [4]. L'unité fonctionnelle correspond à l'hétérodimère RXR-RAR qui se fixe sur une séquence déterminée de la région promotrice des gènes cibles, l'élément de réponse de l'acide rétinoïque (ou RARE pour retinoic acid response element), constitué d'un motif consensus directement répété et espacé de cinq nucléotides (séquence DR5) ([4] et $\left.m / s ~ 1998, n^{\circ} 11, p .1211\right)$. Les deux familles de récepteurs, RAR et RXR, contiennent chacun trois membres, $\alpha$, $\beta$, et $\gamma$, codés par des gènes différents, permettant ainsi de nombreuses combinaisons de cette unité fonctionnelle, ce qui est probablement en partie à l'origine de la diversité des effets biologiques de l'acide rétinoïque dans l'organisme. En l'absence de ligand, le dimère RXR-RAR recrute des co-répresseurs (NCoR, mSin3 et une histone désacétylase) provoquant la compression de la chromatine et donc la répression transcriptionnelle des gènes cibles. L'addition du ligand induit la dissociation du complexe co-répresseur et favorise l'association du dimère RXRRAR avec des co-activateurs. Il s'agit en fait d'un complexe multiprotéique transcriptionnel appelé RANC (retinoic acid-dependent nuclear complex) dans lequel on peut noter la présence de la protéine PML (promyelocytic leukemia), co-activateur dépendant de l'acide rétinoïque, qui n'interagit pas 
directement avec RAR ou RXR, mais avec TIF1 $\alpha$ et CBP, deux partenaires du complexe RANC [5, 6] (figure 1A). Outre les récepteurs nucléaires, deux protéines appelées CRABP I et II (pour cellular retinoic acid binding proteins) lient aussi l'acide rétinoïque dans la cellule [7]. Ces deux protéines de petit poids moléculaire (15 kDa) présentent $75 \%$ d'homologie et sont toutes deux localisées dans le cytoplasme. Elles possèdent des spécificités distinctes vis-à-vis de leurs ligands (l'acide rétinoïque touttrans et l'acide rétinoïque 9-cis) puisque l'affinité de CRABPII pour l'acide rétinoïque tout-trans serait environ trois fois moins grande que celle de CRABPI. CRABPI a une plus large distribution tissulaire que CRABPII qui semble surtout exprimée durant l'embryogenèse, et dans les kératinocytes humains. Leurs fonctions fondamentales communes est de solubiliser, protéger contre les réactions d'oxydo-réduction indépendantes du métabolisme des rétinoïdes, et transporter leurs ligands endogènes respectifs [7]. Les CRABP moduleraient ainsi la voie de signali- sation de l'acide rétinoïque en le séquestrant ou en intervenant sur son catabolisme par son transport vers le réticulum endoplasmique et le cytochrome $\mathrm{P} 450$ associé [7], ce qui permettrait de contrôler la concentration d'acide rétinoïque libre, et donc l'activation des récepteurs nucléaires. Ces protéines cytoplasmiques de liaison aux rétinoïdes n'avaient, jusqu'à très récemment, pas été impliquées dans l'induction d'événements nucléaires.

\section{La leucémie aiguë promyélocytaire: un modèle d'étude de CRABPII}

La leucémie aiguë promyélocytaire est caractérisée par l'accumulation dans la moelle osseuse de cellules tumorales bloquées à l'étape promyélocytaire de la maturation myéloïde. Dans la grande majorité des cas, il existe une translocation chromosomique acquise $t(15 ; 17)$. Celle-ci provoque la synthèse d'une protéine de fusion $\mathrm{PML} / \mathrm{RAR} \alpha$ [8] qui interagit de façon anormale avec les co-répresseurs de la transcription et est responsable du blocage de la différenciation granulo-

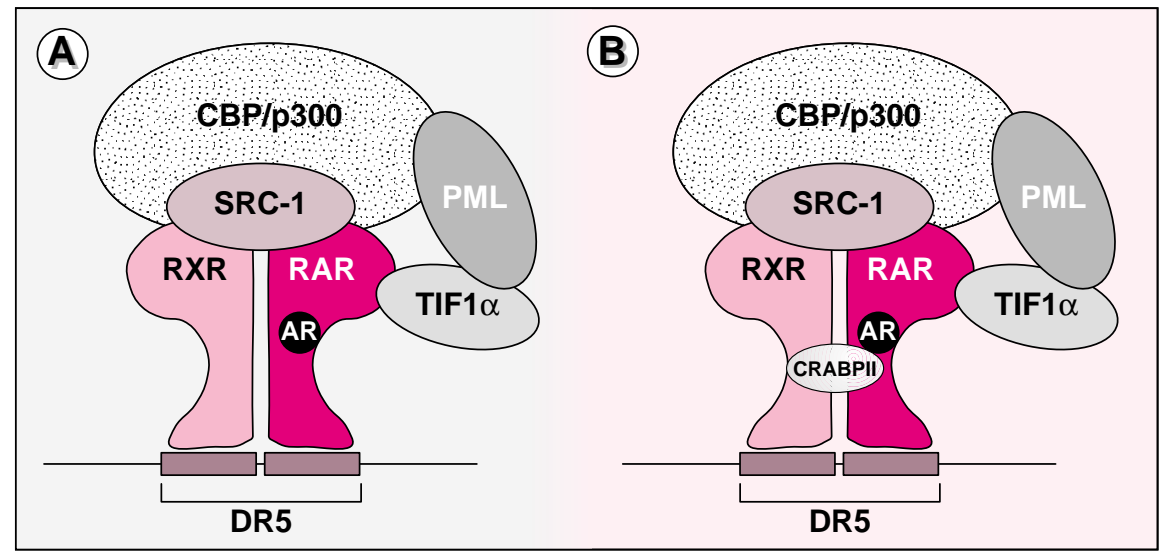

Figure 1. Complexe transcriptionnel des récepteurs de l'acide rétinoïque. A. L'hétérodimère $R X R-R A R$ est fixé sur son élément de réponse DR5. La fixation de l'acide rétinoïque $(A R)$ sur son récepteur induit le recrutement de protéines co-actrivatrices appartenant au complexe RANC (retinoic acid-dependent nuclear complex): $S R C-1$ (steroid receptor coactivator-1), $C B P$ (CREB-binding protein)/p300 qui a une activité histone acetyl transférase, TIF1 $\alpha$ (transcriptional intermediary factor $1 \alpha$ ) et PML (promyelocytic leukemia). PML n'interagit pas directement avec les récepteurs nucléaires $R A R$ et $R X R$, mais avec TIF1 $\alpha$ et CBP. B. Ce schéma peut être maintenant complété avec la protéine CRABPII (cellular retinoic acid binding protein II) qui a la particularité de fixer à la fois le récepteur (RAR ou $R X R)$ et l'acide rétinoïque. Si la fixation du ligand n'est pas nécessaire à l'interaction physique de CRABPII avec les récepteurs, elle semble en revanche indispensable pour augmenter

cytaire. Les cellules leucémiques porteuses de cette translocation sont cependant sensibles à l'acide rétinoïque qui, à des concentrations pharmacologiques, lève ce blocage et induit leur différenciation. Ces importants résultats ont profondément modifié la survie des patients atteints de cette forme de leucémie et le traitement par l'acide rétinoïque a été la première thérapeutique active par son induction de la différenciation de cellules leucémiques $[9,10]$.

Nous avons démontré que le traitement des patients atteints de leucémie aiguë promyélocytaire de type M3 par l'acide rétinoïque tout-trans provoquait l'induction rapide de l'expression de l'allèle normal RAR $\alpha$ dans les cellules leucémiques [11] et, plus tardivement, quand les patients sont en rémission complète, une augmentation de l'expression de CRABPII dans les cellules mononucléées de la moelle osseuse [12]. Le rôle connu des protéines CRABP dans le métabolisme cellulaire de l'acide rétinoïque, laissant penser que le traitement in vivo par des fortes concentrations d'acide rétinoïque tout-trans entrâ̂nait une hypervitaminose A provoquant l'expression et la synthèse des protéines liées au catabolisme de la vitamine $\mathrm{A}$, en particulier CRABPII. Cette hypothèse était renforcée par le fait que l'expression de CRABPII était contrôlée par un élément de réponse pour l'acide rétinoïque. Ceci permettait en outre d'expliquer l'absence d'efficacité de l'acide rétinoïque touttrans comme traitement d'entretien, les patients rechutant pendant ce traitement, ainsi que la survenue d'une résistance secondaire à ce traitement [12, 13]. En fait, des données plus récentes montrent que le rôle de la protéine CRABPII n'est pas restreint à cette fonction de transport et de métabolisme.

\section{CRABPII est un co-facteur des récepteurs nucléaires de l'acide rétinoïque}

Nos résultats ont montré que CRABPII augmente l'activité transcriptionnelle de promoteurs synthétiques ou naturels sensibles à l'action de l'acide rétinoïque [14]. Cet effet a été observé dans des lignées myéloïdes, mais aussi 
dans des cellules non myéloïdes comme des cellules de tumeurs mammaires ou de tératocarcinomes [1416]. Il requiert la présence d'un rétinoïde capable de se lier à la fois aux récepteurs nucléaires de l'acide rétinoïque et à CRABPII, comme l'acide rétinoïque tout-trans et l'acide rétinoïque 9-cis [14]. Ce rôle de la protéine CRABPII dans la transactivation des gènes sensibles à l'acide rétinoïque est en accord avec sa localisation à la fois nucléaire et cytoplasmique. En revanche, CRABPI dont la localisation est uniquement cytoplasmique, au moins dans les cellules hématopoiétiques, n'a aucun effet sur l'activité transcriptionnelle de ces gènes [14]. Cependant, contrairement à certains co-activateurs, CRABPII ne possède pas d'activité transcriptionnelle per se. En fait, nous avons montré qu'elle fait partie du complexe transcriptionnel des récepteurs nucléaires de l'acide rétinoïque.

En effet, des expériences de type « retard sur gel» et d'immunoprécipitation d'extraits nucléaires de cellules hématopoiétiques ou de cellules Cos1 transfectées montrent que CRABPII interagit aussi bien in vivo qu' in vitro avec les deux récepteurs $\mathrm{RXR} \alpha$ et RAR $\alpha$. Nos expériences in vitro montrent que cette interaction est directe, indépendante de la présence ou non du ligand et semble spécifique aux récepteurs de l'acide rétinoïque puisque aucune interaction n'a pu être observée avec d'autres membres de la superfamille des récepteurs stéroïdiens ou d'autres protéines nucléaires. Il apparaît donc que CRABPII fait partie de la classe des co-facteurs des récepteurs des rétinoïdes. dont elle possède certaines spécificités (figure 1B). Cependant, contrairement aux autres coactivateurs, CRABPII est de petite masse moléculaire (16 kd) et ne possède pas la signature spécifique des co-activateurs de la transcription (LXXLL). Sa caractéristique la plus remarquable est de fixer à la fois le récepteur et le ligand, avec de plus une synergie pour la liaison entre le récepteur RAR et CRABPII $[14,16]$. En outre, la présence de la protéine semble faciliter la fixation du complexe multiprotéique RANG sur l'élément de réponse des gènes cibles.
Il apparaît donc que la CRABPII, du fait de ses fonctions multiples, de liaison, de transport et de métabolisme de l'acide rétinoïque, et maintenant sa fonction nucléaire, pourrait jouer un rôle central de coordination de la voie de signalisation des rétinoïdes. Ce mécanisme est observé dans différents types cellulaires, mais il reste à déterminer s'il intervient constamment ou seulement dans certaines conditions cellulaires particulières. L'étude des souris invalidées pour le gène codant pour CRABPII qui, dans des conditions normales, ne présentent pas de phénotype particulier, s'avérera sans doute utile pour préciser le rôle de cette protéine dans la voie de signalisation des rétinoïdes.

\section{Conclusions}

L'intervention des différents facteurs permettant la transactivation des gènes par les récepteurs nucléaires commencent à être mieux connue. Des complexes multiprotéiques impliqués dans le remodelage chromatinien, par une action spécifique sur les nucléosomes, agiraient dans un premier temps, suivis de l'action conjuguée de plusieurs facteurs protéiques possédant une activité histone acétyl transférase. Des complexes multimoléculaires co-activateurs comme DRIP ou TRAP agiraient ensuite au niveau du complexe de préinitiation de la transcription. L'ensemble de ces processus implique de nombreuses interactions protéineprotéine. L'intervention d'une protéine interagissant à la fois avec le récepteur nucléaire et le ligand dans ces complexes protéiques définit un degré supplémentaire de complexité du contrôle de la transcription

\section{RÉFÉRENCES}

1. Xu L, Glass CG, Rosenfeld MG. Coactivator and corepressors complexes in nuclear receptor function. Curr Opin Gene Dev 1999; 9: 140-7.

2. Gelman L, Staels B, Auwerx J. Rôle des co-facteurs transcriptionnels dans la transduction des signaux par les récepteurs nucléaires. Med Sci 1997; 13: 961-70.

3. Lemon BD, Freedman LP. Nuclear receptor cofactors as chromatin remodelers. Curr Opin Genet Dev 1999; 9 : 499-504.

4. Chambon P. A decade of molecular biology of retinoic acid receptors. FASEB $J$ $1996 ; 10: 955-60$.
5. Zhong S, Delva L, Rachez C, et al. A RAdependent, tumour-growth suppressive transcription complex is the target of the PML-RAR $\alpha$ and T18 oncoproteins. Nat Genet 1999; 23: 287-95.

6. Wang ZG, Delva L, Gaboli M, et al. Role of PML in cell growth and the retinoic acid pathway. Science 1998; 279: 1547-51.

7. Napoli JL. Retinoic acid homeostasis. Perspective roles of $\beta$-carotene, retinol, CRBP and CRABP. In : Blomhoff R, ed. Vitamin A in Health and Disease. New York: Marcel Dekker 1994: 135-88.

8. Kalantry S, Delva L, Gaboli M, et al. Gene rearrangements in the molecular pathogenesis of acute promyelocytic leukemia. I Cell Physiol 1997; 173: 288-96.

9. Chomienne C, Fenaux P, Degos L. Retinoid differentiation therapy in promyelocytic leukemia. FASEB J 1996; 10 : 1025-30.

10. Lavau C, Jansen J, Weiss K, Lamond A, Dejean A. Leucémie aiguë promyélocytaire et acide rétinoïque: le paradoxe. Med Sci $1994 ; 10: 817-24$

11. Chomienne C, Balitrand N, Ballerini P, et al. All-trans retinoic acid modulates the retinoic acid receptor alpha in promyelocytic cells. J Clin Invest 1991; 88: 2150-4.

12. Cornic M, Delva L, Guidez F, et al. Induction of retinoic acid binding protein in normal and malignant human myeloid cells by retinoic acid in AML3 patients. Cancer Res 1992; 52: 3329-34.

13. Delva L, Cornic M, Balitrand N, et al. Resistance to all-trans retinoic acid (ATRA) therapy in relapsing acute promyelocytic leukemia: study of in vitro ATRA sensitivity and cellular retinoic acid binding protein levels in leukemic cells. Blood 1993; 82 : 2175-81.

14. Delva L, Bastie JN, Rochette-Egly C, et al. Physical and functional interactions between the cellular retinoic acid binding protein II and the retinoic acid-dependent nuclear complex. Mol Cell Biol 1999; 19: 7158-67.

15. Jing Y, Waxman S, Mira-y-Lopez R. The cellular retinoic acid binding protein II is a positive regulator of retinoic acid signaling in breast cancer cells. Cancer Res 1997; 57 : 1668-72.

16. Dong D, Ruuska SE, Levinthal DJ, et al. Distinct roles for cellular retinoic acid-binding proteins I and II in regulating signaling by retinoic acid.J Biol Chem 1999; 274: 23695-8.

\section{Laurent Delva \\ Jean-Noël Bastie \\ Nicole Balitrand \\ Gilles Despouy \\ Christine Chomienne}

Laboratoire de biologie cellulaire hématopoïétique, EMI 00-03, IUH, Hôpital Saint-Louis, 1, avenue Claude-Vellefaux 75010 Paris, France.

\section{TIRÉS À PART}

\section{Delva.}

